\section{$\underset{\text { \& migrations }}{\text { hommes }}$}

\section{Hommes \& migrations}

Revue française de référence sur les dynamiques

migratoires

$1311 \mid 2015$

Femmes et migrations

\title{
Les allers et retours d'une arpillera chilienne, entre exil et engagement
}

\section{Stéphanie Mahieu}

\section{OpenEdition \\ Journals}

Édition électronique

URL : http://journals.openedition.org/hommesmigrations/3329

DOI : 10.4000/hommesmigrations.3329

ISSN : 2262-3353

Éditeur

Musée national de l'histoire de l'immigration

Édition imprimée

Date de publication : 1 juillet 2015

Pagination : 136-139

ISBN : 978-2-919040-32-2

ISSN : 1142-852X

Référence électronique

Stéphanie Mahieu, «Les allers et retours d'une arpillera chilienne, entre exil et engagement », Hommes \& migrations [En ligne], 1311 | 2015, mis en ligne le 09 février 2016, consulté le 15 septembre 2020. URL : http://journals.openedition.org/hommesmigrations/3329 


\title{
COLLECTIONS
}

\section{LES ALLERS-RETOURS D'UNE ARPILLERA CHILIENNE, ENTRE EXIL ET ENGAGEMENT}

\section{L'ITINÉRAIRE DE MARIA REYES}

\begin{abstract}
par STÉPHANIE MAHIEU, conservateure du patrimoine, responsable des collections ethnologiques, Musée national de l'histoire de l'immigration.
\end{abstract}

es donateurs de la Galerie des dons du Musée

national de l'histoire de l'immigration sont aussi des femmes, migrantes ou descendantes de migrants, qui souhaitent donner une visibilité à leur histoire familiale dans le cadre d'une institution patrimoniale capable de la relayer vers des publics plus larges.

"J'aurais pu vous offrir autre chose, quelque chose de plus léger comme un oiseau en paille, mais cette arpillera est porteuse de beaucoup de choses, non seulement de mon histoire familiale, mais aussi d'une période plutôt noire du Chili... Enfin pas uniquement noire, parce qu'il y a aussi le côté Unité populaire', les idéaux, très présents dans cette arpillera. C'est une manière de montrer que, même si le Chili n'a pas encore cicatrisé toutes ses blessures, il y a un témoignage direct, avec cet objet, de ce qu'a pu être le Chili des années 1970, pour moi elle représente vraiment cet idéal auquel je suis attachée" (Maria Reyes).
L'histoire de Maria Reyes est celle des nombreux hijos del exilio², les enfants des exilés chiliens ayant fui la dictature du général Pinochet dans les années $1970^{3}$, et dont beaucoup furent accueillis en France ${ }^{4}$. Cette génération, qui a reçu l'exil en héritage, est aujourd'hui adulte et enracinée en France. Cependant, beaucoup ont grandi avec le rêve du retour au pays. L'histoire de la famille de Maria Reyes est singulière : ses parents ont tenté un tel retour au moment de la transition démocratique dans les années 1990. Elle a passé ses années d'adolescence au Chili, avant que toute la famille ne revienne finalement vivre en France. Elle souhaite donner au Musée national de l'histoire de l'immigration un objet hautement symbolique de sa double identité, française et chilienne, et des allers et retours transatlantiques de sa famille : une arpillera, seul objet qu'elle a emmené avec elle lors du retour en France de sa famille à la fin des années 1990, sur laquelle figurent les mots trabajo,

1. L'Unité populaire, en espagnol Unidad Popular (UP), est le résultat d'un accord politique entre les partis de centre-gauche et de la gauche du Chili ayant pour but l'élection de Salvador Allende à la présidence de la République, le 4 septembre 1970. 2. Pour reprendre le titre de la série Hijos del exilio d'Eric Facon, photographe au Bar Floréal. L'ensemble des photos et des textes de la série a été publié dans un ouvrage intitulé Hijos del exilio / Enfants de l'exil, photographies d'Éric Facon et texte de Diego Olivares, éditions Créaphis, 2013. 3. Sur l'histoire de l'exil chilien en France, on consultera le numéro spécial d'Hommes \& Migrations, $n^{\circ}$ 1305, janvier-février-mars 2014 : “L’Exil chilien en France”. Et sur la question spécifique de l’héritage de l'exil des descendants d'exilés politiques chiliens, voir Fanny Jedlicki : "Venceremos, Pinocho y Las Empanadas, Grandir en exil à l'ombre du retour", pp. 33-39. 4. Sur l'accueil des réfugiés chiliens en France, voir l'article de Marie-Christine Volovitch-Tavares, "L’accueil en France des réfugiés après le 11 septembre 1973”, in Hommes \& Migrations, n 1305, janvier-février-mars 2014 , pp. 49-56. 
justicia y libertad (travail, justice et liberté). Les arpilleras sont des courtepointes colorées tissées par des femmes de détenus politiques et de chômeurs pendant la dictature du général Pinochet (1973-1990)5. Maria Reyes, née en 1982 en France de parents chiliens, enseigne l'espagnol dans un lycée de Boulogne. Elle a pris connaissance de la Galerie des dons sur Internet et a souhaité faire don de cet objet important dans son histoire personnelle et familiale, qui se trouvait auparavant dans son salon: "Je me suis dit que cétait une manière généreuse de transmettre de façon concrète quelque chose qui est abstrait. L'immigration en elle-même, on la porte en soi, et c'est difficile d'expliquer avec des mots sensés ce que c'est, ce qu'on ressent au quotidien. Pour moi, l'immigration se représente mieux par les objets ou avec de l'art, de la musique, mais je ne suis pas artiste, je ne sais pas dessiner, je ne sais pas jouer de la musique. Chez moi, ce sont des objets qui me rappellent le Chili. Ils font partie de mon identité... ce n'est pas de la décoration, c'est moi!"

\section{Du Chili vers la France : un exil en héritage}

"Je suis la fille de José Reyes et de Eugenia Razeto. Mon père est d'origine chilienne, et ma mère est une Chilienne issue de la deuxième génération d'Italiens. Mes parents sont des exilés politiques qui ont fui le régime de Pinochet. L'histoire de ma famille est une histoire d'allers et de retours. Leur histoire est basée sur le militantisme, c'est-à-dire qu'ils se sont rencontrés ici en France parce qu'ils étaient militants. Je suis fière d'être leur fille, car ils incarnent pour moi des valeurs qu'ils ont su me transmettre, ainsi qu'à mon frère et à ma sceur..." De ses premières années en banlieue parisienne, elle ne garde que des souvenir très flous: "De mon enfance, c'est assez étrange, je n'ai pas beaucoup de souvenirs, j'ai l'impression que je suis née au Chili, à l'âge de dix ans. C'est assez déroutant, on en parle parfois avec ma sceur jumelle, et il n'y a rien qui ressort. Toute ma famille est chilienne, même si j'ai quelques parents éloignés du côté de ma mère en Italie. Je n'ai pas ressenti le manque de famille avant de l'avoir connue, mais je n'ai pas de souvenirs exceptionnels de Noël, ou de quelque chose, avant de revenir au Chili." Ce départ pour le Chili a eu lieu en 1992, quatre ans après le suffrage de 1988 et deux ans après le retour de la démocratie ${ }^{6}$. Maria Reyes était alors âgée de dix ans.

\section{Une vie santiaguina}

Le père de Maria, qui travaillait à la Banque de France, avait obtenu le droit de prendre une disponibilité de quatre ans. Elle a peu de souvenirs de la façon dont ses parents lui ont annoncé ce "retour au pays" : "Je me rappelle que je n'étais pas très contente parce que je me sentais bien en France, mais c'est vrai que j'étais assez curieuse de connaitre la famille. J'avais une idée du Chili... Pour moi, le Chili c'était vraiment le tiers-monde. Je me souviens que lorsque jeécrivais à mes cousines, je me disais: 'Les pauvres, elles doivent vraiment vivre dans la misère.' Mais, une fois sur place, le premier décalage a été de dire : 'Au fond, ils sont comme nous, ils vivent ailleurs mais ils ne sont pas dans la misère, ils ne sont pas malheureux."'

La famille s'installe à Providencia, un quartier résidentiel de Santiago, dans une maison appartenant à son grand-père maternel. Sa mère obtient un poste de professeur de philosophie, et à un moment ils évoquent même l'idée de s'établir de manière définitive. Pourtant, le retour au pays s'avère plus compliqué que prévu : "C'est-à-dire qu'ily a eu un décalage....je crois qu'ils sont rentrés là-bas avec beaucoup d'illusions, peut-être aussi avec les idéaux de l'Unidad Popular, ils se sont 
“Travail, justice, liberté." La arpillera de Maria Reyes.

(c) Sébastien Morin.

rendu compte que finalement l'adaptation à leur pays était plus difficile que ce qu'ils avaient imaginé, ce n'était plus trop leur pays, dans la mesure où toutce quiétaitfacileà trouver avant (du travail, etc.) devenait maintenant compliqué. Une fois qu'on sort du système et qu'on n'a plus d'activité professionnelle, c'est compliqué. Ma mère est revenue en tant que professeur de philosophie, et elle a arrêté assez rapidement. Avec trois enfants, ce métier, ce n'est pas comme en France, on ne travaille pas dix-huit heures par semaine, mais plutôt quarante heures, plus les copies et tout le reste..." Pendant les années chiliennes, les trois enfants de la famille sont scolarisés à l'Alliance française de Santiago, grâce à des bourses du gouvernement français. Plus que la langue (la scolarité étant bilingue), c'est plutôt la différence de conception du monde qui a marqué Maria, en particulier l'enseignement de la colonisation : "J'ai trouvé que la colonisation elle-même, cétait très compliqué. J'ai dû mettre plusieurs mois à comprendre pourquoi cela fonctionnait comme cela, avec un roi en Espagne, comment quelqu'un qui était si loin pouvait régner. Cela me semblait invraisemblable, c'est ce rapport au monde différent qui a été la plus grande difficulté, plus que la langue." Peu à peu, l'adolescente s'adapte pourtant à l'école. 


\section{Un nouveau voyage transatlantique : retour définitif en France?}

Finalement, au terme des quatre ans de disponibilité qu'avait obtenus son père, ses parents ont pris la décision de retourner vivre en France. Cela n'a pas été une décision simple, car tout le monde savait que ce départ-là du Chili serait sans doute le dernier. La jeune femme n'a pourtant pas le souvenir qu'ils en aient parlé longtemps avant: "Ils n'ont jamais dit que cela n'allait pas. Je crois que cétait plus de la pudeur pour nous protéger, cela a été assez rapide... C'était plus ou moins comme lorsque nous sommes partis au Chili, cela s'est fait rapidement, peut-être six mois avant, et je me souviens que, lorsque je devais partir, j'en parlais à ma meilleure amie, et elle me disait : 'Dis-le, on va te faire une fête (j'étais en cinquième), il faut qu'on fête ton départ.' Mais pour moi c'était quelque chose de tellement triste de partir, de laisser ma famille, que je ne l'ai dit à personne. J'avais un sentiment un peu de honte, de devoir me justifier de pourquoi je partais, de ne pas avoir forcément de motifs à dire. C'est plus facile de dire : 'Je suis venue pour connaitre la famille', que de repartir sans raison à l'autre bout du monde. Bon, avec le temps j'ai compris..." Toute la famille a repris le cours de sa vie en France et les trois enfants ont suivi des études universitaires et sont aujourd'hui dans une vie professionnelle active.

En 1992 comme en 1996, chaque enfant n'avait le droit de prendre avec lui que peu d'objets. À son départ de France, la jeune fille avait emmené une peluche avec elle. À son retour, quatre ans plus tard, elle emporte l'arpillera dont elle fait don au Musée : “À l'époque, j'avais compris que c'était quelque chose d'important, mais je n'avais pas encore conscience de la dictature, de tout ce qui s'était passé. J'avais à peine conscience de Pinochet. Pour moi, c'était un vieillard très méchant qui rendait mes parents malheureux, et je m'étais dit, du haut de mes dix ans: 'Je vais le rencontrer ce Pinochet, et je vais lui parler, je vais lui dire d'arrêter de rendre mes parents malheureux, parce que mes parents sont gentils!'Donc,j'avais vraiment une idée très lointaine de la dictature, mais j'avais compris que cette arpillera était un objet important, et les couleurs sont magnifiques, cela m'a toujours captivée. Tout comme la peluche représentait la France, cette arpillera symbolisait le Chili, cétait un bout de Chili que je pouvais emmener avec moi." La force symbolique tout autant que le côté visuellement fort des arpilleras n'a pas échappé à plusieurs musées, qui ont souligné l'importance de la fabrication de ces objets pendant les années sombres de la dictature ${ }^{7}$ et leur aspect polysémique, entre art populaire, message de protestation et document d'archive. De nombreuses arpilleras sont exposées dans leurs galeries ou sur leurs sites Internet. Elles ont ainsi été présentées dans une exposition en 2014-2015 au V\&A Museum de Londres intitulée Disobedient Objects', et font l'objet d'une exposition virtuelle sur le site du Royal Alberta Museum ${ }^{9}$ d'Edmonton au Canada, ainsi que sur celui du William Benton Museum of Art aux États-Unis ${ }^{10}$.

Avec la proposition de don de Maria Reyes, le Musée national de l'histoire de l'immigration prolonge un tel intérêt et peut présenter aux visiteurs de la Galerie des dons un objet personnel venu de l'autre bout du monde, porteur d'un message de lutte et d'espérance. 